\title{
Investigation on mechanical behaviour of ECAPed 2A12 aluminium alloy
}

\author{
Cun-xian Wang ${ }^{1}$, Chao Zhang ${ }^{1}$, Lei Wang ${ }^{1}$, Zhong-bin Tang ${ }^{1,2}$, and Tao Suo ${ }^{1,2, \text { a }}$ \\ ${ }^{1}$ School of Aeronautics, Northwestern Polytechnical University, 710072 Xi' an, China \\ ${ }^{2}$ Fundamental Science on Aircraft Structural Mechanics and Strength Laboratory, Northwestern Polytechnical University, \\ 710072 Xi'an, China
}

\begin{abstract}
In the present work, the uniaxial compressive behavior of aluminum alloy processed by equal channel angular pressing (ECAP) for 1-8 passes are investigated experimentally under both quasi-static and dynamic loading conditions via an electronic universal testing machine with a maximum load capacity of $10 \mathrm{KN}$ and the split Hopkinson pressure bar (SHPB). The strain hardening rate as well as strain rate sensitivity the ECAPed with different passes have been determined and compared with annealed coarse grained counterpart. The experimental results show a continuously increase of both flow stress and strain rate sensitivity for the aluminum alloy subjected to ECAP process as the pressing pass number increasing. It is proposed that the reduction in grain size plays an important role in the enhancement of flow stress and strain rate sensitivity. However, the strain hardening rate of the ECAPed materials decreases remarkably. Meanwhile, compressive experiments at elevated temperatures indicate the temperature sensitivity of the material increases as the grain size is refined into fine grain regime. Based on thermal activation theory, it is proposed that the enhanced temperature and strain rate sensitivity of ECAPed aluminum alloy can be related to the reduction in activation volume due to grain refinement.
\end{abstract}

\section{Introduction}

In the past few years, ultra fine-grained/nanocrystalline materials show many unusual physical and mechanical properties due to grain refinement and the larger proportion of the grain boundary, which attracted the attentions of many domestic and foreign scholars. A lot of research results indicated that grain refinement can lead to the increase of both strength and the change of deformation mechanism [1-4]. For example, it is observed that the tensile strength of ECAPed 7075 aluminium alloy can reach as high as 677 MPA while the ECAPed 2024 aluminium alloy can also reach $618 \mathrm{MPa}$; (Zheng et al. [5]). There are also many literatures pay attention on the strain rate sensitivity of the ultra fine-grained/nanocrystalline materials. Experimental studies show that the strain rate sensitivity of the facecentred cubic crystal metals decreases with the grain size increases, while the body-centered cubic on the contrary, with the reduction in grain size [6].

Strain hardening is also an important mechanical property of materials, which reflects the relations between the flow stress and the flow strain. From the microscopic mechanism, the material microstructure hinder the movement of the movable dislocations, then the strain hardening is generated. The temperature, strain rate will affect the strain hardening properties of aluminium alloys. For ultrafine-grain aluminium fabricated by ECAP, the biggest factor of the strain hardening properties is the high dislocation density [7]. But the investigations of the effect on strain hardening by temperature at the high strain rate are still relatively rare.

\footnotetext{
${ }^{\text {a }}$ Corresponding author: suotao@nwpu.edu.cn
}

Previous studies are mainly concentrated on the mechanical properties of aluminum alloys in the quasistatic condition, few works pay attention to the dynamic mechanical behavior of aluminum alloy. The limited works indicates that the microstructure changes will not only cause a significant improvement of the mechanical properties in the quasi-static condition, dynamic mechanical properties are also observed to change accordingly [8].

Although the research on the mechanical behaviour of fine-grained aluminium alloy has been made in the past few years, there still remain many issues especially the coupling effects of the strain rate and temperature on the mechanical behaviour of the fine grained materials. The primary aim of this work is to investigate the coupling effects of the strain rate and temperature on compressive behaviour of ECAPed-2A12 aluminium alloy. The strain rate and temperature sensitivities as well as the temperature sensitivity of the material are analyzed based on the experimental results.

\section{Material preparation and experimental procedure}

In the present work, the commercial 2A12 aluminium alloy was selected as the experimental materials. The raw rods with the dimension of $9.8 \mathrm{~mm} \times 9.8 \mathrm{~mm} \times 60 \mathrm{~mm}$ were annealed for two hours at the temperature of $573 \mathrm{~K}$. After that, the ECAP method was employed using a hydraulic machine at a speed of $\sim 15 \mathrm{~mm} / \mathrm{min}$ at $473 \mathrm{~K}$ to produce fine grained material. The ECAP die used in this work has the geometry of an inner corner angle of $\Phi=90^{\circ}$ and an outer angle of $\psi=0^{\circ}$ (see in Fig. 1). To achieve ultrafine

This is an Open Access article distributed under the terms of the Creative Commons Attribution License 4.0, which permits unrestricted use, distribution, and reproduction in any medium, provided the original work is properly cited. 


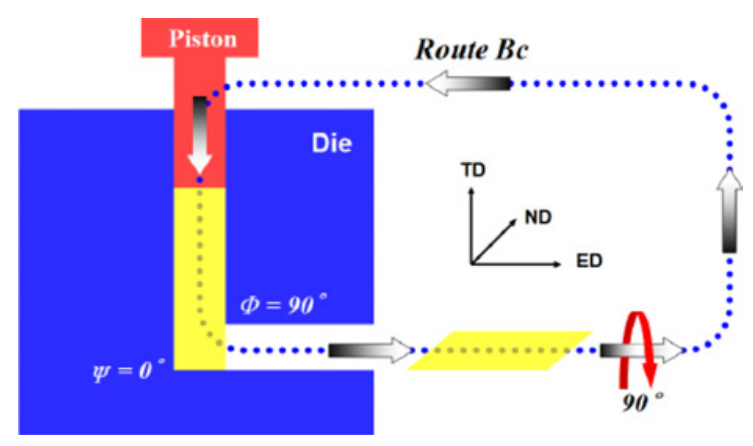

Figure 1. Schematic sketch of ECAP.

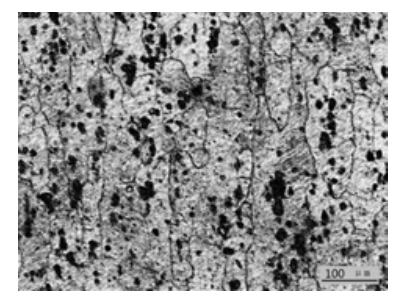

(a)

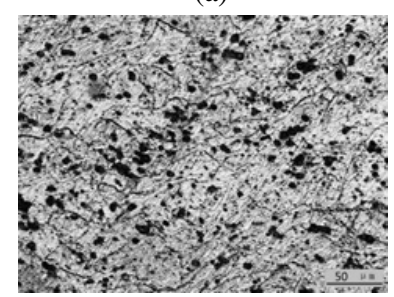

(c)

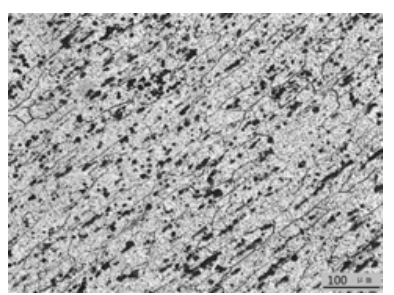

(b)

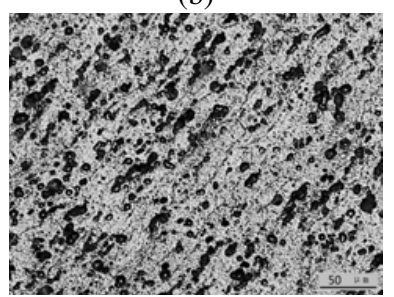

(d)
Figure 2. Micrographs of ECAPed-2A12 with different passes (a) 0 pass, (b) 1 pass, (c) 4 passes, (d) 8 passes.

grained microstructure, each specimen was pressed 8 passes using route $\mathrm{Bc}$.

Quasi-static compressive experiments were performed using an electronic universal testing machine with a maximum load capacity of $10 \mathrm{KN}$. The split Hopkinson pressure bar (SHPB) was employed for high strain rate experiments. According to the one dimensional elastic stress wave theory, the dynamic strain, stress and strain rate of tested specimen can be calculated respectively [9]. The specimens for both quasi-static and dynamic compressive tests were taken from the center part of the ECAPed rods and machined to cylinders with dimensions of $\Phi 4 \times 4 \mathrm{~mm}$. The loading direction was parallel to the ECAPed rod axis. During the tests, the strain rates were controlled from $10^{-3}$ to $10^{3} \% \mathrm{~s}$

\section{Results and discussion}

\subsection{Microstructure of ECAPed-2A12}

Figure 2 gives the metallographic micrographs of 1 pass, 4 pass, 8 pass ECAP aluminium alloy as well as the annealed alloy. It can be seen from the pictures that the grain size of annealed 2A12 aluminium alloy is about $100 \mu \mathrm{m}$. However, most of grains are elongated after the first ECAP process and the large grains transform gradually to sub-grains accompanied by small angle grain boundaries (see in Fig. 2(b)). After the

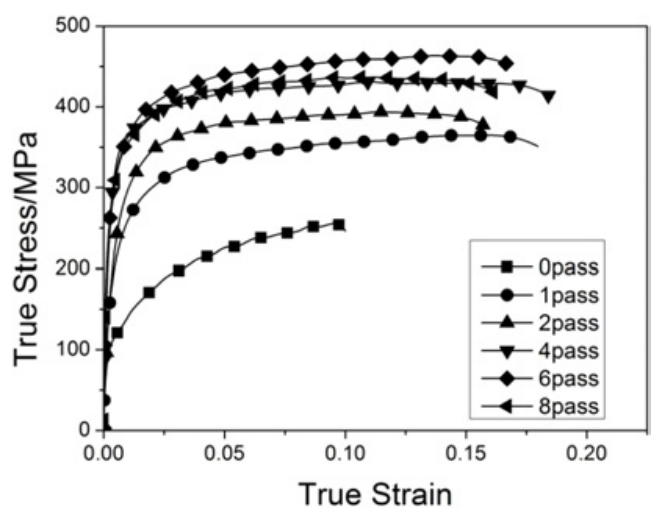

Figure 3. The true stress versus true strain curves of ECAP-2A12 aluminum alloy at the strain rate of $0.001 / \mathrm{s}$

further passes (see in Fig. 2(c)), those elongated grains were broken into fine grains with the average grain size of $3 \mu \mathrm{m}$. Comparison of the microstructure of 4 pass ECAPed-2A12 with that of 8 pass alloy indicates that the additional 4 passes give almost no further grain refinement to the aluminium alloy. However, more homogenous distribution of equaxial grains is believed to be achieved by the further plastic deformation $[8,10]$. From the figures, many $\mathrm{Al}_{2} \mathrm{CuMg}$ compounds with the length $0.8 \mu \mathrm{m}$ and width $0.4 \mu \mathrm{m}$ can also be found in $\alpha$-Al the substrate. The further plastic deformation does not change the size of these $\mathrm{Al}_{2} \mathrm{CuMg}$ compounds but the distribution of them seems more uniform in the 8pass ECAPed-2A12 if compared with that in annealed alloy.

\subsection{Strength enhancement after ECAP process}

Figure 3 shows the true stress versus true strain curves of ECAPed-2A12 aluminium alloys with different passes under quasi-static loading. It can be seen that the yield stress and flow stress of the material increases with pass number until reach the maximum values after 6 passes. If comparing the flow stress of 8 pass ECAPed-2A12 alloy with that of 6 pass, there is no significant increment. This is in good agreement with the microstructural observation that the grain size of ECAPed-2A12 reaches the minimum value after 4 passes. The fact that the ECAP-2A12 aluminium alloy displays higher strength as the grain size is reduced into ultrafine grain regime can be explained by the remarkable increment of dislocation density after sever plastic deformation as well as the classical HallPetch relationship from which the flow stress of metals is inversely proportional to square root of grain size.

\subsection{Strain rate effects}

Figure 4 shows true stress-strain curves of ECAPed-2A12 aluminium alloy deformed at $293 \mathrm{~K}$ under both quasistatic and dynamic loading conditions. For comparison, the true stress-strain curves of the annealed coarse grained aluminum alloy at different strain rates are also shown in Fig. 4(a). It can be easily found that the ECAPed-2A12 aluminium alloy show almost elastic-ideal plastic behaviour at quasi-static strain rate while for the annealed coarse-grained aluminium alloy remarkable 


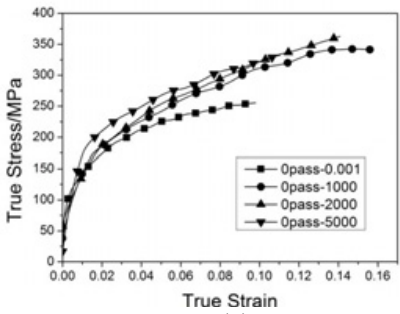

(a)

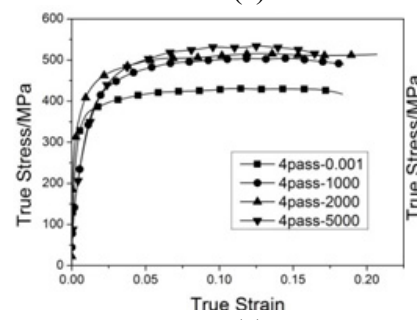

(c)

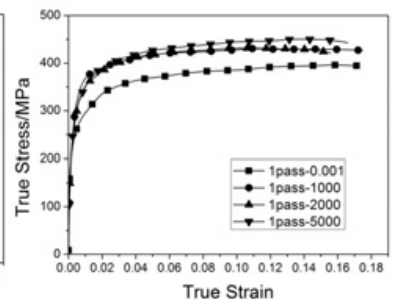

(b)

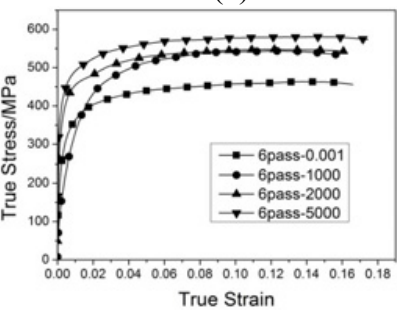

(d)

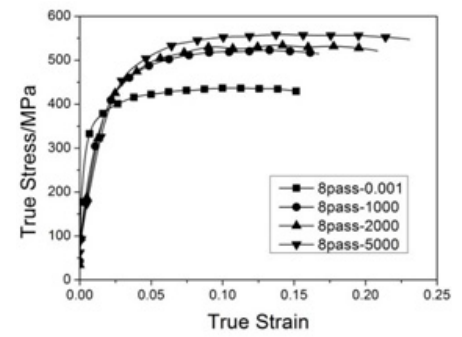

(e)

Figure 4. True stress-strain curves of ECAPed-2A12 aluminum alloy at different strain rates (a) 0 pass, (b) 1 pass, (c) 4 passes, (d) 6 passes, (e) 8 passes.

strain-hardening can be observed. Wang et al. [7] also carried out systemic study on the strain hardening behaviour of the material after grain refinement and the results showed that the strain hardening behaviour of the material decreased rapidly as the grains is refined. This is in good consistent with the results of the present work. The reason for the poor strain hardening capability is believed to be that the internal dislocation density of the material is very high after severe plastic deformation which leads to higher dislocation dynamic recovery rate than coarse-grained materials. During the initial stage of plastic deformation, a large number of dislocations generate from dislocation sources which subsequently interact with grain or sub-grain boundaries, causing high strain hardening rate. However, the enhanced dynamic recovery process makes the balance between generation and annihilation of dislocations quickly, leading to a steady-state dislocation density and hence the material undergoes a long deformation stage with slight variation in strain hardening rate $[3,7,11]$. It should be pointed out that with increasing strain rate, the ECAPed-2A12 aluminium alloys with different passes show strain hardening behaviour more or less, and the strain hardening rate increases with strain rate.

As can be seen from the true stress-strain curves of ECAP-2A12 aluminium alloy, the change of strain rate affects the strength of the material. Some studies have shown that the grain refinement will lead to the changes of strain rate sensitivity (SRS) $[6,7,12]$. In general, the strain

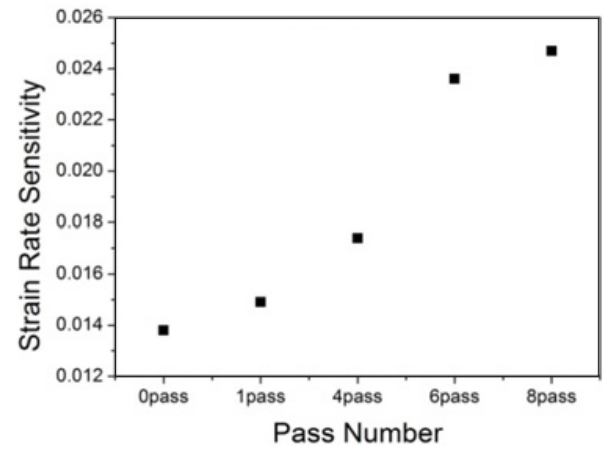

Figure 5. Strain rate sensitivity of ECAPed-2A12 with different passes.

rate sensitivity generally increases with grain refinement on the face-centred cubic (FCC) metals while strain rate sensitivity decreases with grain refinement on the body-centred cubic (BCC) metals [6]. It is believed that higher strain rate is not conducive to the slip spread continuity between the grains and may cause large stress concentration in the vicinity of grain boundaries easily which will increase the deformation resistance [13]. The dependence of flow stress on the applied strain rate is usually described quantitatively by a coefficient named strain rate sensitivity (SRS) factor $m$, which can be expressed as

$$
m=\left.\frac{\partial \ln \sigma}{\partial \ln \dot{\varepsilon}}\right|_{\varepsilon}
$$

where $\sigma$ and $\varepsilon$ are flow stress and strain respectively. According to the formula, the values of SRS can be determined approximately by using log-log flow stress versus strain rate plots at a certain strain from which $\mathrm{m}$ was derived as the slope of a linear regression fit $[6,14]$. It should be point out that the value of $m$ is a function of applied strain according to the definition. In this work, the flow stresses corresponding to the fixed true strain of 0.07 are employed to calculate the strain rate sensitivity factor.

Figure 5 shows the SRS factor for ECAPed-2A12 aluminium alloy after different passes. It can be seen from the figure that the strain rate sensitivity of ECAPed2A12 aluminium alloy increases remarkably with the pass number until to 6 passes. After that, slight increment of SRS is found as the pass number increases from 6 to 8. As mentioned above, although the latter passes may not change the subgrain size and perhaps the overall dislocation density, the misorientation among the subgrains is expected to be changed and the dislocations may be rearranged. Consequently, the dislocation density in the vicinities of grain boundaries can be rather high, which may lead to a larger contribution to the thermally activated deformation processes (higher SRS value) $[8,10]$.

Since plastic deformation of metals is thermal activated process, for studying the physical mechanism of plastic deformation of crystals, an effective method is thermalactivation analysis. Employing the concept of thermalactivation theory, the strain rate sensitivity factor can also be given by

$$
m=\frac{\sqrt{3} k T}{\sigma V^{*}}
$$




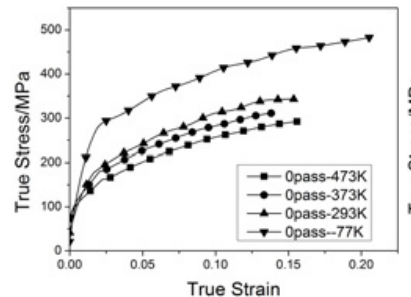

(a)

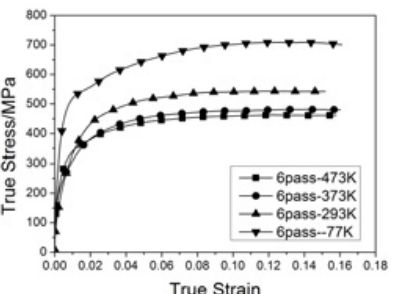

(b)
Figure 6. Stress-strain curves of annealed and 6 pass ECAPed$2 \mathrm{~A} 12$ alloy at strain rate of 1000/s and at different temperatures.

where $\dot{\varepsilon}$ is applied strain rate, $T$ is the absolute temperature and $V^{*}$ is thermal activation volume. It can be seen from the equation that the strain rate sensitivity can be affected by the combined effects of stress and activated volume at fixed temperature. We all know that the fully annealed metals with FCC structure usually have a large activation volume, i.e. $\sim 10^{2}-10^{3} \mathrm{~b} 3$, and the dominant rate-controlling process is the intersection of moving dislocations cutting through forest dislocations [13]. While for fine grained aluminium, the activation volume is believed to be at least one order lower than that of its coarse grained counterpart although the thermally activated forest dislocation intersections tend to occur in the vicinity of grain boundaries or/at the grain boundaries where the density of dislocations is supposed to be higher [8]. Therefore, it's easy to understand that the strain rate sensitivity of ECAPed-2a12 alloy can be enhanced since the enhancement in flow stress is limited if compared with the annealed aluminium alloy.

\subsection{Temperature effects}

Figure 6 shows the true stress-strain curves of annealed coarse-grained aluminium alloy and 6 pass ECAPed2A12 aluminium alloy at strain rate of 1000/s and at different temperatures. It can be seen that with the decreasing of test temperature, flow stresses of the two materials significantly increase. For annealed coarse-grained aluminium alloy, strain hardening is more obvious. However, the flow stress of 6pass ECAPed-2A12 aluminium alloy increases rapidly only at the beginning of plastic deformation. With the rising temperature, the strain hardening rate of annealed alloy keeps almost unchanged. While for the ECAPed alloy, it shows remarkably strain hardening behaviour at the cryogenic temperature $(77 \mathrm{~K})$. It is believed that the low temperature can suppress the dynamic recovery of dislocations which cause obvious strain hardening behaviour [15]. The fact that materials can regain the ability of strain hardening at cryogenic temperature has been proved by Wang [7] as a strategy to achieve larger uniform tensile deformation.

Figure 7 shows the flow stress variation with temperature of the ECAPed-2A12 aluminium alloy and the annealed coarse-grained aluminum alloy at the strain rate of $10^{3} / \mathrm{s}$ at the fixed strain of $7 \%$. It can be seen from the Fig. 7 that the flow stress of the material tends to increase with the decreasing temperature. Meanwhile, we can clearly see that as the temperature rises the flow stress of the material with finer grains changes more

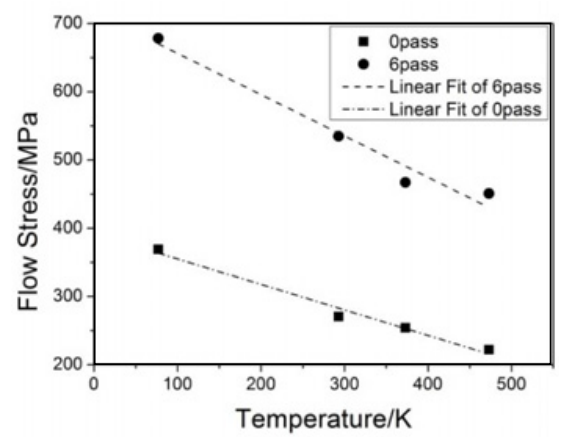

Figure 7. Variation of flow stress of the ECAPed and the annealed coarse-grained aluminium alloy at fixed strain of $7 \%$ with temperature.

significantly, indicating the flow stress is more sensitive to temperature for the ECAPed-2A12 aluminium alloy.

In order to analyze the influence on the temperature sensitivity of flow stress by the grain size changes, the temperature sensitivity factor can be defined as [15]:

$$
S_{T}=\left(\frac{\partial \sigma}{\partial T}\right)_{\varepsilon} .
$$

It can be seen from Eq. (3) that the temperature sensitivity changes with strain. Therefore, the corresponding strain must be indicated when using the formula. The slope of the linear fit line in Fig. 7 is the estimation of the temperature sensitivity factor of the material. The temperature sensitivity factor of the ECAPed-2A12 aluminium alloy and the annealed coarse-grained aluminum alloy can be calculated to be -0.51 and -0.83 respectively where "-" means that the flow stress decreases with the increasing temperature. This indicates that the temperature sensitivity increases obviously with the grain refinement. It is believed that the unusually small activation volume in NC or UFG FCC metals implies a stronger temperature dependence of the yield and flow stress. Also based on thermal-activation theory, the temperature sensitivity factor can be expressed as [16]

$$
\frac{\partial \sigma}{\partial T}=-\sqrt{3} K \ln \frac{\dot{\varepsilon}_{0}}{\dot{\varepsilon}} / V^{*}
$$

The above equation does indicate that at fixed strain rate the $S_{\mathrm{T}}$ depends on the evolution of activation volume. For UFG materials with FCC crystal structure, the activation volume has been proven to decrease with the reduction in grain size. Therefore, the smaller the grain size, the higher the temperature sensitivity of flow stresses is expected. This conclusion is in good agreement with the experimental data. Since the pre-exponential strain rate, $\dot{\varepsilon}_{0}$, is of the order of $10^{6}-10^{8} / \mathrm{s}$ [17] which is several orders of magnitude higher than aare expectedin rates in the present tests, values of temperature sensitivity is expected to be negative according to Eq. (4). This indicates the reduction in flow stress with increasing temperature.

\section{Conclusions}

In the present work, we have carried out uniaxial compressive experiments on ECAPed-2A12 aluminium 
alloy fabricated by the ECAP method over a wide range of temperatures and strain rates. It can be seen from the experimental results that the yield stress of the 2A12 aluminium alloy increases with ECAP pass number. However, the yield stress of ECAPed-Al peaks after six passes and changes slightly in further passes. Due to fine grain aluminum alloys own great dynamic recovery ability, their strain hardening capacity decreases remarkably. The strain rate sensitive factor of ECAPed-2A12 aluminium alloy increases with pass number. It is believed that the reduction in grain size as well as increase of dislocation density after SPD process contribute to the enhanced strain rate sensitivity. If comparing with the annealed coarse grained aluminium, the temperature sensitivity of ECAPed-2A12 aluminium alloys is relatively high. It is believed that the enhanced temperature sensitivity of finegrained aluminium alloy can be related to reduction in activated volume.

This work was performed under the financial support from the National Science Foundation of China (grant Nos. 11272267, 11372206 and 11102168) and 111 Project (grant No. B07050) of Northwestern Polytechnical University.

\section{References}

[1] R.Z. Valiev, T.G. Langdon, Prog. Mater Sci. 51, 881-981 (2006)
[2] J.C.M. Li. Mechanical Properties of Nanocrystalline Materials (Pan Stanford Publishing, New York, 2011)

[3] M.A. Meyers, A. Mishra, D.J. Benson, Prog. Mater. Sci. 51, 427-556 (2006)

[4] R.Z. Valiev, R.K. Islamgaliev, I.V. Alexandrov, Prog. Mater. Sci. 45, 103-189 (2000)

[5] L.J. Zheng, C.Q. Chen, T.T. Zhou, Mater. Characterization 49, 455-461 (2002)

[6] Q. Wei, J. Mat. Sci. 42, 1709-1727 (2007)

[7] Y.M. Wang, E. Ma, Mat. Sci. Eng. A 375, 46-52 (2004)

[8] T. Suo, Y.Z. Chen, Y.L. Li, C.X. Wang, X.L. Fan, Mat. Sci. Eng. A 560, 545-551 (2013)

[9] H. Kolsky, Proc. Phys. Soc. B 62, 676-700 (1949)

[10] F.H. Dalla Torre, E.V. Pereloma, C.H.J. Davies,. Acta Mater. 54, 1135-1146 (2006)

[11] L. Zhu, J. Lu, Inter. J. Plasticity 30-31, 166-184 (2012)

[12] C.C. Koch. Scripta Mater. 49, 657-662 (2003)

[13] D. Jia, K.T. Ramesh, E. Ma, Acta Mater. 51, 34953509 (2003)

[14] G.T. Gray III, T.C. Lowe, C.M. Cady, R.Z. Valiev, I.V. Aleksandrov, Nanostructured Mater. 9, 477-480 (1997)

[15] T. Suo, Y. Li, F. Zhao, X. Fan, W. Guo, Mech Mater, 61, 1-10 (2013)

[16] G. Malygin, Physics Solid State 49, 1013-1033 (2007)

[17] H. Conrad, D. Yang, J Electron Mater. 31, 304 (2002) 